\title{
La teoría de los hemisferios cerebrales y el método Botetano
}

\author{
The theory of the cerebral hemispheres and method botetano
}

\author{
César Botetano C. ${ }^{1}$ \\ Uniliersidad Nacional Mayor de San Marcos, Lima, Perú \\ (RECIBIDO 03/04/2014, ACEPTADo 20/06/2014)
}

\begin{abstract}
RESUMEN
Hemos trabajado con más de 4 mil niños en Perú, Bolivia y México con excelentes resultados en el área del cálculo mental, más específicamente en el aprendizaje de las tablas de multiplicar hasta el 20 en un plazo de no más de 10 clases. Y encontramos en la Teoría de los Hemisferios Cerebrales el marco teórico necesario que nos da el soporte necesario para poder explicar un aprendizaje que para muchos profesores del nivel primario resulta sorprendente.

Los pobres resultados en las pruebas de PISA en el área de las matemáticas entre los niños de nuestro país nos llevaron a buscar un método alternativo al método tradicional basado en la memorización. En una búsqueda que nos llevó varios años, finalmente encontramos que el juego, el razonamiento, la competencia, el desafío y el aprendizaje basado en elementos sensoriales del niño nos dio este sorprendente resultado. Hemos sido capaces de captar el interés de los niños con juegos y cuentos que apelaban directamente a su imaginación y esto se basa precisamente en la teoría de los hemisferios cerebrales. Este artículo se refiere al encuentro de la enseñanza intensiva basada en el trabajo práctico y una teoría que le da el apoyo necesario.
\end{abstract}

Palabras clave: Aprendizaje de las matemáticas, hemisferios cerebrales, Método tradicional

\section{ABSTRACT}

In order to explain the great results obtained by children in mental calculation, I am forced to find an answer in the field of learning theories. I consider that the Theory of Cerebral Hemispheres gives us the explanation that I was looking for. After getting many interesting results with more than 4000 children in countries like Peru, Bolivia and Mexico related to the learning of mental arithmetic in general and multiplication tables in particular, we find that the theory of the cerebral hemispheres were able to give us the necessary theoretical background we needed to explain these results that many consider very interesting.

The poor results in PISA tests in the area of mathematics among children of our country led us to seek an alternative method to the traditional method based on memorization. In a search that took us several years, I finally found that gaming, reasoning, competition, challenge

1 Profesor-investigador del aprendizaje de las matemáticas. Estudió Psicología en la UNMSM. Email:metodobotetano@ gmail.com 
LA TEORIA DE LOS HEMISFERIOS CEREBRALES Y EL MÉTODO BOTETANO

and learning based on the child's sensorial elements gave us this amazing results. We were able to capture the interest of children with games and stories that appealed directly to their imagination and this is precisely based on the Theory of the Cerebral Hemispheres. This article refers to the encounter of intensive teaching based on practical work and a theory that gives it the necessary support.

Keywords: Learning of mathematics, cerebral hemispheres, traditional method

\section{PRESENTACIÓN}

Empezamos hace más de diez años trabajando de manera específica sobre una nueva forma de aprender a multiplicar con niños entre 7 y 12 años. Nuestro método difería de una manera sustancial con el método tradicional basado en la memoria y la repetición. Nosotros incidimos de una manera fundamental en el razonamiento, el juego, el desafío y la competencia para lograr el referido aprendizaje. Dejamos de lado el uso del lápiz, papel y pizarra y trabajamos en el campo del cálculo mental. Durante estos diez años hemos trabajado con más de 4 mil niños en Perú, Bolivia y México, de diferente condición social. Nuestros resultados han sido altamente satisfactorios. Durante esta década hemos venido desarrollando los principios de nuestro método, basado como hemos dicho en el razonamiento y en el juego. No obstante, no alcanzábamos a comprender del todo la razón por la que los niños alcanzaban a aprender de una manera tan efectiva y espectacular. Al dar con la teoría de los hemisferios cerebrales, por fin pudimos encontrar una explicación satisfactoria. Este encuentro entre nuestro método y la referida teoría de los hemisferios cerebrales motiva el presente ensayo.

\section{PLANTEAMIENTO DEL PROBLEMA}

La educación es un medio poderoso para lograr igualdad de oportunidades que permitan avanzar hacia una sociedad más justa y democrática. Así, la política educativa es fundamental para alcanzar este fin. Los últimos resultados dados a conocer en 1997 por el TIMSS (Tercer Estudio Internacional de Matemáticas y Ciencias), de un estudio realizado en más de 45 países en el curso 94/95, considera que "en todos los países del mundo las matemáticas son un papel importante del currículum escolar, y se consideran materias esenciales para la formación de los jóvenes (...) y para desarrollar en el individuo hábitos de razonamiento riguroso y crítico. Los resultados del rendimiento español en los cursos de $7^{\circ}$ y $8^{\circ}$ de E.G.B. (en los que se efectuaron las pruebas) son, en ambos casos, inferiores al rendimiento medio internacional".

Las matemáticas es la materia más prestigiada socialmente, se le da valor predictivo sobre las capacidades del individuo y prevalece el "mito de las matemáticas" en relación con los niveles de inteligencia, y a la expectativas de bienestar futuro (Guerrero Ojeda, 1989: p. 57). Malén Aznárez (1997: p. 78) califica las matemáticas como "la materia que ha sido para generaciones de españoles, y aún lo es para muchos, el coco y pesadilla de sus años de estudiante. Una pesadilla 
irremediable porque los niños aprenden desde bien pequeños que la primera nota por la que se interesan sus padres es por la de matemáticas". Siguen Dienes, "son muy pocos los profesores de matemáticas que se encuentren satisfechos del modo en que transcurre su enseñanza y la mayoría de niños que sienten antipatía por las matemáticas -antipatía que aumenta con la edad- encuentran dificultades casi insuperables en las cuestiones más sencillas. La mayor parte de los niños nunca llega a comprender la significación real de los conceptos matemáticos, o se convierten en técnicos en el arte de manejar complicados símbolos. La mayoría desiste de comprender las imposibles situaciones de las exigencias de las matemáticas escolares. La actitud más corriente es esforzarse en aprobar un examen, tras lo cual nadie dedica a las matemáticas ni un pensamiento más.

Es reconocida la importancia de las matemáticas, su capacidad de servir a la tecnología, la industria, la ciencia. La responsabilidad de los profesores es considerar cuidadosamente el estilo de su enseñanza. En los últimos años distintos autores han concedido un papel importante a las estrategias que permiten al sujeto la toma de conciencia de la actividad mental, concediéndole gran relevancia a los aspectos cognitivos. Sin embargo, se presta menor atención a la actividad emocional, su introducción hace valer el rol significativo que tienen las emociones como facilitador o debilitador del aprendizaje y a que la calidad emocional en la clase ejerza una influencia significativa.

En el mismo artículo, Gómez Chacón (1997, p. 5) expone la alfabetización emocional, que "engloba habilidades tales como el control de los impulsos y fobias en relación con la asignatura, control que permite desarrollar la necesaria atención para que se logre el aprendizaje, la autoconciencia, la motivación, el entusiasmo, la perseverancia, la empatía, la agilidad mental, etc."

Uno de los grandes problemas que plantea la enseñanza de las matemáticas (y de la enseñanza en general) queda reflejado en la rueda de Dyer:

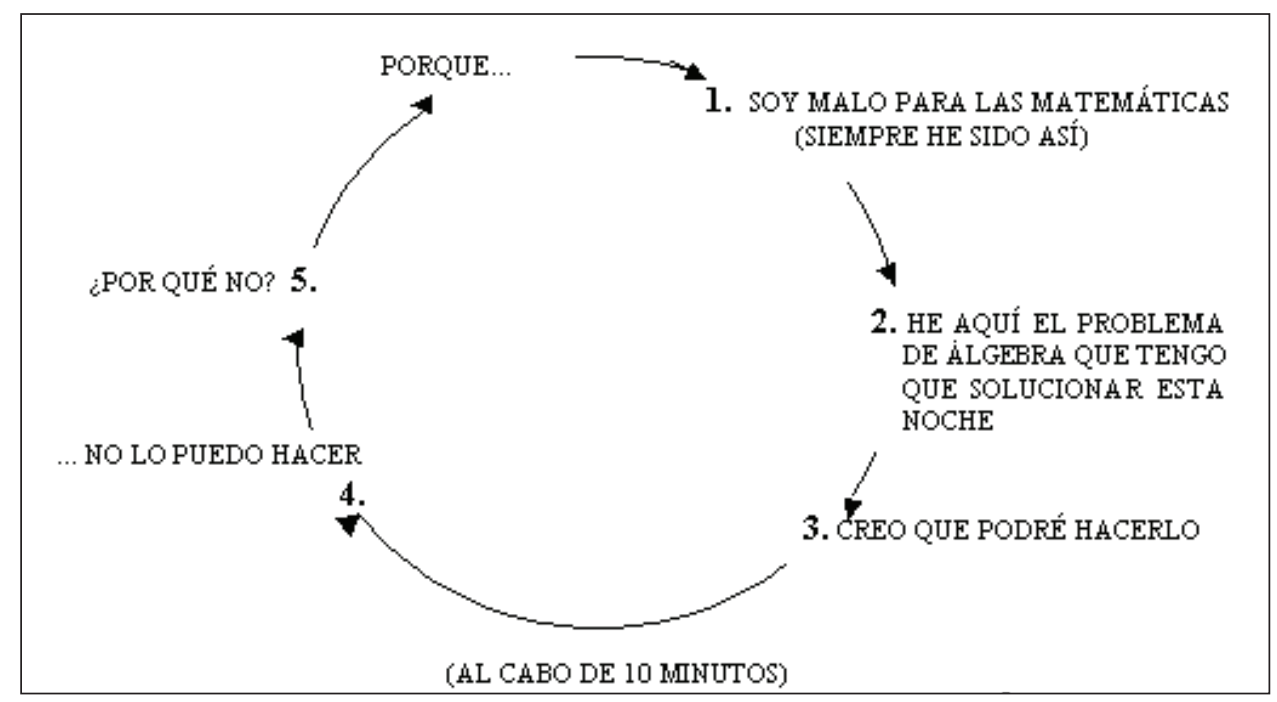


La memoria no basta para la matemática, es necesaria la comprensión. La repetición de ejercicios es buena solo si los estudiantes saben lo que están haciendo. El problema del aprendizaje de las matemáticas se plantea por el bajísimo rendimiento escolar de los niños, específicamente en la multiplicación, operación fundamental, pues es una suma abreviada e inversa de la división. Casi todo el aprendizaje en nuestro sistema educativo se basa principalmente en la memorización. El alumno repite al pie de la letra el concepto para aprobar dicha materia. No interesa si lo ha comprendido, internalizado y pueda repetir con sus propias palabras. Si la inteligencia es la capacidad de poder descubrir las conexiones internas de los fenómenos que estudiamos, descubriendo las relaciones causales, lejos están los estudiantes de poder hallar y/o descubrir tales relaciones en las materias que estudian. La multiplicación aprendida por la repetición de una tabla, sin significación, es odiada por semejante práctica. Esta es la razón fundamental de los pobrísimos resultados que se obtienen de la manera tradicional como se enseña a multiplicar.

\section{HACIA UN NUEVO PARADIGMA DEL APRENDIZAJE DE LA MATEMÁTICA: EL MÉTODO BOTETANO}

\section{El método en acción}

El método Botetano se desarrolló como un cambio de paradigma de la enseñanza de la matemática a los niños. Un hecho ilustra este cambio, en relación con niños de 5 años en México, del Kinder-3. La profesora dijo que los niños podían contar hasta el número 10 y demandado bastante esfuerzo. Le planteé el reto de hacerles contar hasta el número 40 o más en un par de clases. Ella sonrío incrédula. Puse el método en acción: en vez de la didáctica de la profesora de mostrar los números y hacerles repetir de manera oral y escrita, apele a la desbordante imaginación de los niños: les pedí que dejaran sus pupitres y se sentaran junto conmigo en el suelo. Luego, que imaginaran que estábamos en un barco pirata y todos éramos piratas bajo el mando de un terrible pirata llamado Barba Negra, el cual podía castigar a cualquier miembro de la tripulación a la más mínima falta que cometieran. Les dije que este terrible personaje no sabía leer ni contar, que nos había dejado abierto los cofres de monedas (fichas de colores) y de joyas (botones de colores), así que podíamos repartirnos una parte del tesoro, pero era necesario contarlo para hacerlo. Llegado a este punto, a los niños les brillaban los ojos por semejante reto. Pedí que me ayudaran a contar. De inmediato los niños empezaron a hacerlo. Y contaban: "uno, dos, tres... ocho, nueve, diez" y luego continuaban: "diez y uno, diez y dos, diez y tres". En ese momento procedí a corregirlos de una manera amigable y les enseñé la manera correcta. En menos de media hora los niños llegaban con facilidad al 40 y más. Cuando la profesora retornó, quedó asombrada.

\section{Características del método Botetano}

Se puede describir el método determinando las características de su praxis, en su accionar en el proceso mismo de enseñanza - aprendizaje. 
1) No se escribe nada. Todo el proceso en una primera etapa es mental. Se enfatiza el razonamiento por encima de todo. Por consiguiente, el niño no basa su aprendizaje en la memorización. Cuando aprende las tablas de multiplicación memorizándolas, si se olvida de alguna respuesta, no tiene forma de salir del problema. En cambio, cuando aprende a razonar para obtener las respuesta, tiene la capacidad de corregirse si se equivoca o de obtener la respuesta si se olvida. Cuando el niño ha logrado entender cómo se llega a la respuesta adecuada, la práctica intensa le da rapidez y muchos creen que ha memorizado las tablas, pero no es así. En este caso, la práctica hace al maestro.

2) El cálculo mental tiene la ventaja de dar capacidad de razonamiento mental, que se logra ejercitando con técnicas de cálculo mental, las mismas que plantean maneras cortas, rápidas y diversas para llegar al resultado. Así, se supera la forma mecánica y única planteada para solucionar sumas, restas o multiplicaciones. Se privilegia el aspecto del razonamiento por encima de todo. Se le reconoce múltiples virtudes al cálculo mental, entre las que podemos citar solo algunas como: a) genera una comprensión más profunda de la estructura de los números y de sus propiedades, b) desarrolla formas creativas e ingeniosas para manejar los números, c) impulsa el desarrollo de un pensamiento creativo e independiente y d) desarrolla la capacidad de establecer cálculos estimados.

3) Incentivando a que el niño desarrolle su capacidad de visualización, enfatiza la comprensión de lo que se enseña. Se parte de ejemplos concretos y prácticos, luego a una práctica rápida e intensiva de ellos. Finalmente, se deja de lado los apoyos visuales, para lograr que el niño pueda visualizar y realizar los ejercicios apoyándose únicamente en su mente.

4) No se agrupa a los niños como si todos fueran iguales, como si todos tuviesen las mismas capacidades de aprendizaje. La enseñanza con el método es con un máximo de 15 alumnos por grupo y con una profesora y una asistente. De manera que las clases son casi personalizadas. Tratamos cada caso, cada niño, respetando profundamente su individualidad y personalidad.

5) Considera la importancia de contextualizar cada capítulo o tema que se enseña. Así, las clases captan el interés y participación plena. Nuestra experiencia y estudios al respecto nos demuestran que haciendo uso, por ejemplo, de cuentos y narraciones que ayudan a contextualizar y despertar el interés de los niños es la mejor manera de introducir al niño a cada nuevo tema o concepto. Esto es de suma importancia. Se logra de manera extraordinaria que el niño se involucre, participe, y busque sus propias respuestas por ensayo y error, sin que se las proporcione el maestro, para que se esfuerce en lograrlas.

6) En lo posible se recurre al desarrollo histórico de cada punto, de manera que el niño trate de entender el porqué de la importancia y necesidad de cada actividad, sea esta la suma o la multiplicación. 
7) Demostrándole que las matemáticas están presentes en su vida diaria y que no están circunscritas solo a su salón de clase,vincula de manera activa lo que aprende el niño con su entorno diario y cotidiano. Puede utilizarlas en cosas tan frecuentes como pagar las cuentas o calcular los gastos cuando sale de compras.

8) Una característica resaltante del sistema es darse en un ambiente de juego, competencia y desafío entre los niños que participan en su aprendizaje. La razón de esto se basa en que esa es precisamente la naturaleza de los niños.

9) El método se aplica en módulos con contenidos de aprendizaje de las matemáticas por edades: Módulo 1: de 3 a 4 años, objetivo: agrupar, clasificar, seriar. Contar del 1 al 20. Módulo 2: de 4 a 5 años, objetivo: todo lo anterior y además contar y sumar del 1 al 50. Módulo 3: de 5 a 6 años, objetivo: todo lo anterior y además sumar y restar del 1 al 100. Multiplicar hasta la tabla del 6. Módulo 4: de 6 años para adelante, objetivo: todo lo anterior y además sumar y restar mentalmente cantidades de 3 cifras. Multiplicar hasta la tabla del 15 e incluso hasta la tabla del 20. Los resultados que se obtienen son simplemente asombrosos.

\section{El método Botetano un nuevo sistema de enseñanza}

El proceso comenzó originalmente como Multilúdica, luego fue Phi Master Kid, y finalmente se consolidó como método Botetano. Se parte de diseñar de una manera puntual el aprendizaje de las tablas de multiplicar: sin memorizarlas y usando el razonamiento, el juego y la competencia. Estas características se aplican no solo al aprendizaje de las tablas de multiplicar, sino también al aprendizaje de la suma, resta, quebrados, etc. El universo original de alumnos que se daba entre 7 y 12 años, se amplia hasta incluir a los niños desde los 3 años. Lo que nos llevó a trabajar en la base misma del aprendizaje numérico desde su inicio.

En México comprendí que había desarrollado un nuevo sistema de enseñanza de las matemáticas, alternativo al sistema tradicional que abusa de la memorización, da poca importancia al proceso de razonamiento, escinde el aprendizaje de los conceptos matemáticos con su aplicación en la vida diaria, descuida el dominio conceptual de los temas a cambio de una exagerada práctica operativa, sin que el estudiante tenga claro para qué le servirá lo que aprende.Con el nuevo sistema creado se descubre el enorme potencial matemático en cada niño de inteligencia normal, que permanece latente e inexplotado debido a la manera tradicional de acercarlo a las matemáticas. Se reproduce de manera abreviada cómo naturalmente el hombre ha desarrollado sus habilidades matemáticas, vinculando el aprendizaje a su aplicación práctica, empezando como un proceso mental, luego de entender y dominar los conceptos con la parte operativa, dejando para más adelante su consolidación de manera escrita. Estimulando en los niños el razonamiento sobre la memoria, generando un ambiente de juego, desafío y competencia entre ellos, los resultados son simplemente espectaculares. Los resultados que hemos obtenido en muchos pilotos y talleres llevados a cabo nos dan la confianza que vamos por el camino correcto. 
César Botetano

\section{III.LA TEORÍA DE LOS HEMISFERIOS CEREBRALES Y EL APREN- DIZAJE}

\section{La teoría de los hemisferios cerebrales}

Explicar la eficacia del método Botetano, de sus resultados espectaculares en el aprendizaje de las matemáticas con los niños, me motivó a realizar investigaciones exploratorias en el campo de las teorías del aprendizaje. La explicación plena se logra con la teoría de los hemisferios cerebrales.

La teoría de los hemisferios cerebrales explica la estructura y función del cerebro humano, en dos hemisferios, cada uno con cuatro lóbulos, conectados entre sí por el corpus callosum. Cada hemisferio procesa la información que recibe de distinta manera o hay distintas formas de pensamiento asociadas con cada hemisferio. El hemisferio lógico, el izquierdo (HI), procesa la información de manera secuencial y lineal. El hemisferio lógico forma la imagen del todo a partir de las partes y es el que se ocupa de analizar los detalles. El hemisferio lógico piensa en palabras y en números. El hemisferio holístico, el derecho (HD), procesa la información de manera global, partiendo del todo para entender las distintas partes que componen ese todo. El hemisferio holístico es intuitivo no lógico, piensa en imágenes y sentimientos.

Aunque no siempre el hemisferio lógico se corresponde con el hemisferio izquierdo ni el holístico con el derecho, en un principio se pensó que así era, por lo que con frecuencia se habla de alumnos hemisferio izquierdo (o alumnos analíticos) y alumnos hemisferio derecho (o alumnos globales). Para poder aprender bien, es necesario usar los dos hemisferios, pero la mayoría de nosotros tendemos a usar uno más que el otro. El que tendamos a usar más una manera de pensar que otra determina nuestras habilidades cognitivas ya que cada manera de pensar está asociada con distintas habilidades en función del modo de pensamiento que prefieran.

R. Zenhausern (1979) diseñó un test (26 ítems) que discrimina la preferencia hemisférica cerebral, entre el derecho (espacial/visual) y el izquierdo (verbal) y lo denominó $<<$ Diferential Hemispheric Activation Instrument $>>$. El HI es verbal y el HD, espacial/visual. Los estudios sobre el cerebro señalan que, cuando procesa la información, funciona con un doble proceso integrado; por una parte, un proceso analítico o proporcional bajo el control prioritario (aunque no exclusivo) del hemisferio izquierdo, y por otra un proceso sintético u oposicional bajo el control prioritario (aunque no absoluto) del hemisferio derecho. Entonces, el HI se relaciona con un proceso analítico o proporcional; el HD se relaciona con un proceso sintético u oposicional. Esta conexión del HI y HD es lo que caracteriza el sistema Botetano.

\section{La educación basada en el cerebro}

El cerebro está íntimamente involucrado con todo lo que los educadores y los alumnos realizan en la escuela. Cualquier desconexión es receta para la frustración 
y un potencial desastre. La educación basada en el cerebro es el entrecruzamiento de varias estrategias basadas en los principios derivados de nuestra comprensión del cerebro, se centra en el conocimiento de por qué se ha de usar una u otra estrategia. El modelo de educación basada en el cerebro es interdisciplinario. En esta dirección, Antonio Damasio dice: "La relación entre los sistemas cerebrales, la cognición compleja y el comportamiento solo pueden ser explicados satisfactoriamente a través de una mezcla integral de teorías y hechos relacionados con todos los niveles organizativos del sistema nervioso".

El cerebro humano puede hacer crecer nuevas neuronas que se hacen funcionales. Las neuronas nuevas están correlacionadas con la memoria, el estado de ánimo y el aprendizaje. Asimismo, las condiciones sociales influencian nuestro cerebro de formas que antes no conocíamos. El descubrimiento de neuronas espejo por parte de Giacomo Rizzolatti sugiere que hay reciprocidad imitativa en nuestro cerebro. La habilidad del cerebro para reorganizarse a sí mismo a través de lo que se conoce como neuroplasticidad es enorme. Ahora sabemos de la capacidad de nuestros genes para responder a impulsos crónicos o agudos del entorno. Este descubrimiento ha echado luces sobre nuevas oportunidades de cambio para nuestros estudiantes.

Las evaluaciones constantes llevan a que algunos educadores eliminen los períodos de descanso y de juego o la educación física, propios del HD. El descubrimiento de que los diversos ambientes alteran nuestro cerebro es muy importante. Si nuestro cerebro está involucrado en todo lo que hacemos, la siguiente pregunta es: ¿nuestro cerebro es fijo o maleable?, ¿la experiencia le da forma? Gran cantidad de evidencias muestran que nuestro cerebro se altera por las experiencias cotidianas, tales como aprender a leer, aprender nuevo vocabulario, estudiar para las pruebas. El cerebro está involucrado en todo lo que hacemos en la escuela; los educadores que comprendan esto tomarán decisiones al respecto. En tanto que muchas escuelas se encuentran reduciendo la actividad física, un amplio grupo de estudios ha vinculado la actividad física con la cognición. Ahora, agreguemos la perspectiva de la neurociencia. Esta revela información que otras disciplinas no pueden revelar. Por ejemplo, sabemos que el ejercicio está altamente correlacionado con la neurogénesis, la producción de nuevas células cerebrales, Los educadores deberían decir: "Estos estudios sugieren que $\mathrm{X}, \mathrm{Y}$ o $\mathrm{Z}$ puede ser cierto, en relación con el cerebro. Hecha esta aclaración, tal vez sea atinado decir que, bajo estas circunstancias, se pueden utilizar tales estrategias en la escuela". La educación basada en el cerebro no es una panacea ni una magia para resolver todos los problemas de la educación. Aún no se trata de un programa, modelo o paquete instruccional para que lo sigan las escuelas. La discusión sobre cómo mejorar el aprendizaje de los estudiantes debe extenderse de axones y dendritas, para contemplar un panorama más amplio, es decir, afirmar que nuestro cerebro evoluciona con todo lo que hacemos en la escuela. El cerebro es el asunto más importante a explorar debido a que afecta cada estrategia, acción, comportamiento y política en nuestra escuela. 


\section{Neurociencia aplicada a la educación}

Tradicionalmente, el sistema educativo occidental ha presentado la tendencia a privilegiar en las aulas, de manera casi excluyente, una modalidad de transmisión del conocimiento compatible con las funciones cerebrales propias o predominantes del HI, en detrimento del gran potencial propio del HD. Sin embargo, a la luz de los nuevos descubrimientos de la neurociencia, no es posible ignorar los enormes beneficios que pueden representar para el estudiante la implementación de estrategias educacionales que incluyan la activación neuronal de ambos hemisferios, fomentando la estimulación del cerebro de manera global, posibilitando una mayor y mejor asimilación de los contenidos a aprender. Así, se propone en el campo educativo tener en cuenta las características funcionales del cerebro humano, de modo que se desarrollen técnicas y estrategias de trasmisión del conocimiento compatibles con la biología del aprendizaje.

La presentación diversificada del contenido de cualquier asignatura o materia a aprender dentro del claustro educativo siempre será más efectiva si implica la activación neuronal de ambos hemisferios cerebrales. Así, serían eficaces las estrategias de enseñanza utilizando la imaginación, la metáfora, la experiencia de primera mano: directa o vivencial; el arte, la música y la apelación a los cinco canales sensoriales propios del ser humano, más allá de la vista y el oído tradicionalmente priorizados. Se propone, siempre que sea posible, la incorporación del tacto, gusto y olfato; múltiples entradas que doten de significado al aprendizaje y lo conviertan en un acto vivencial que potencie los procesos de fijación de la memoria. Sobre esta base se postula algunos lineamientos de aplicación práctica en los procesos de enseñanza y aprendizaje:

Trabajar en una modalidad simultánea o paralela. Por ejemplo, cuando un alumno lee un libro o un manual sobre el antiguo imperio romano, se activa el hemisferio cerebral izquierdo gracias a su modalidad lógico-verbal, lo que implica un menor procesamiento de información en una unidad de tiempo, en comparación al hemisferio contralateral. Proveer al alumno de experiencias sensoriales enriquecidas favorece la activación del hemisferio cerebral derecho, que posee la ventaja adicional de procesar una mayor cantidad de ítems en forma simultánea, a través de múltiples canales sensoriales. La utilización de videos podría representar una ganancia adicional; insume mucho menos tiempo ver una película que leer el guión de ella.

Ver lo holístico y global. Un error pedagógico es presentar las asignaturas de forma aislada o inconexa. Por ejemplo, la historia argentina contemporánea podría ser enseñada asociada a un marco global de la historia universal. Promoviendo un mejor entendimiento de los diferentes acontecimientos locales entrelazados y en interacción dinámica con el resto de los sucesos mundiales.

Seguir una lógica analógica. Este punto implica la trasmisión de conceptos más o menos complejos o abstractos, apelando a la metáfora y a todas las formas de imágenes visuales. Por ejemplo, se podrían desarrollar cómics o historietas sobre la campaña de Napoleón en Europa, exhibir películas británicas con y sin subtitulado para un correcto modelado de la fonética inglesa. 
Permitir la comprensión de los hechos a través de la vivencia. El cerebro humano, en tanto órgano plástico, permeable, experimenta cambios físicos reales de manera constante y como consecuencia de una permanente interacción con el medio ambiente. Las experiencias de vida esculpen literalmente el cerebro propiciando el desarrollo y crecimiento del árbol dendrítico entre las neuronas, que son las células cerebrales implicadas en todos los procesos cognitivos y pilares y sustrato biológico del aprendizaje.

Las diferentes vivencias cotidianas promueven la creación de redes neurales nuevas y refuerzan circuitos neurales preexistentes. La arquitectura del cerebro de un niño o adolescente en desarrollo cambia; se establecen nuevas conexiones entre las neuronas de la corteza prefrontal, que es la parte pensante del cerebro, cuando existe la posibilidad de interacción con un medio ambiente rico y variado en estímulos. Un aprendizaje basado en la vivencia directa o de "primera mano", que trascienda la limitada capacidad pedagógica de un libro de texto o una clase oral, es imprescindible para el desarrollo de la inteligencia práctica y compatible con los conocimientos actuales sobre el funcionamiento del cerebro humano. La utilización de huesos reales o modelos, por ejemplo, en una clase de anatomía, que se puedan ver y tocar y superponer en su propio cuerpo, es un valor agregado decisivo, superior a la mera lectura sobre en un libro de texto. Asimismo, experimentos reales en un laboratorio de ciencias para asignaturas como física o química, una visita a una laguna o bosque que sortee las paredes del aula en materias como geografía o biología, etc.

Los circuitos neurales se construyen a través de la afectividad primaria. El sistema atencional, sobre el que se apoya el aprendizaje, con asiento anatómico en la corteza prefrontal del cerebro, se encuentra conectado por una densa carretera de fibras nerviosas con estructuras del sistema límbico, como el cíngulo anterior y la amígdala, responsables de la motivación, el libre albedrío y el procesamiento del componente emocional de los estímulos. De lo expuesto, se desprende que atención y emoción son dos aspectos de la cognición en permanente interacción, o es el estado de ánimo, el humor y las emociones quienes comandan la atención. Un clima emocional positivo dentro del aula puede erigirse como uno de los mayores facilitadores para el aprendizaje del alumno. La emotividad positiva debe edificarse sobre la confianza y respeto, en un ambiente seguro y predecible para todos; con un sistema de normas y reglas claras, estipuladas por consenso entre los interesados, y así se minimice la posibilidad de conflictos, el estrés que envenena todo proceso de aprendizaje eficaz. La solidaridad, el cuidado mutuo, el trabajo en equipo, donde todos ganan o todos pierden, son estrategias promotoras del sosiego necesario dentro del aula que abona el camino para la permeabilidad cerebral y receptividad del alumno. Las hormonas del estrés, como la noradrenalina y el cortisol, segregadas por el cerebro en respuesta a situaciones de adversidad o sobrecarga tensional, afectan los procesos de consolidación de la memoria y bloquean la corteza prefrontal, impidiendo dirigir y focalizar la atención hacia las experiencias de aprendizaje, alterando la capacidad de resolución inteligente de problemas y enturbiando notablemente habilidades esenciales para la toma de decisiones. 
El responsable de los procesos creativos y el arte en general. Es posible crear experiencias de aprendizaje compatibles con el funcionamiento del hemisferio cerebral derecho, mediante la simulación o representación actoral de sucesos históricos, el dibujo, la educación plástica, la música e incluso la lengua y las letras, con el desarrollo de novelas cuya trama aparezca enmarcada dentro de un contexto sociohistórico específico que se pretende enseñar, o cuentos y fábulas que ilustren o metaforicen contenidos abstractos, etc.

Conclusión. El ser humano llega al mundo con un cerebro parcialmente desarrollado. La posibilidad de que el cerebro continúe creciendo y desarrollándose después del nacimiento es por la adquisición de vivencias que son la base de la inteligencia práctica. Los circuitos neurales de la corteza prefrontal continúan su ramificación y expansión con seguridad casi hasta la tercera década de vida.

El sistema educativo occidental ha privilegiado el desarrollo del hemisferio cerebral izquierdo. De esta manera, se pierde de vista que la vida se desarrolla por la mediación de un cerebro que posee dos hemisferios, que funciona de manera global inserto y en interacción con un mundo real, vivencial y sensorial. Por consiguiente, resulta imprescindible fomentar el desarrollo del hemisferio cerebral derecho. La revisión de los viejos planes de estudio, como punto de partida para un nuevo abordaje de los procesos de enseñanza y aprendizaje que actúen en la misma modalidad de funcionamiento global del cerebro humano, es el desafío para los educadores de este nuevo siglo.

\section{Las teorías de los de los hemisferios y los estilos de aprendizaje}

Despins (1985) diferencia las tareas prioritarias emprendidas por los hemisferios cerebrales, determinando cuatro estilos de aprendizaje. Dos ligados al HD: Estilo 1: intuitivo y divergente y Estilo 4: estilo experimentador, sintético y creativo. Y dos estilos controlados por el HI: Estilo 2: estilo analítico y formal y Estilo 3: estilo práctico y convergente. A partir de los estilos de aprendizaje, se pueden organizar las estrategias didácticas que activen el cerebro del niño. Una enseñanza equilibrada debe tener en cuenta los cuatro estilos de aprendizaje y las actividades cerebrales. Hay que ayudar al niño a que sea capaz de recurrir a los cuatro estilos de aprendizaje, superando la polarización en alguno de ellos, que redundará en el futuro en una menor capacidad de aprendizaje. Despins aclara que una descripción de estilos de aprendizaje debe entenderse de forma correcta y comprensiva. Es la descripción de grados y no comportamientos estancos. Si se afirma que un alumno es intuitivo-divergente, significa que en él predominan estos caracteres específicos, aunque pueda también ser capaz de análisis, conceptualización y síntesis.

El cerebro humano se caracteriza por su capacidad de relacionar y asociar la información, pero no todos seguimos el mismo procedimiento. La manera de organizar la información afecta nuestro estilo de aprendizaje. Cada hemisferio procesa la información que recibe de distinta manera; según como organiza la información, se distingue entre alumnos HD y alumnos HI. El hemisferio lógico, normalmente el izquierdo, procesa la información de manera secuencial y lineal. El hemisferio lógico forma la imagen del todo a partir de las partes y es el que 
se ocupa de analizar los detalles. El hemisferio lógico piensa en palabras y en números, es decir, contiene la capacidad para la matemática y para leer y escribir. Este hemisferio emplea un estilo de pensamiento convergente para obtener nueva información al usar datos ya disponibles, formando nuevas ideas o datos convencionalmente aceptables. El hemisferio holístico, normalmente el derecho, procesa la información de manera global, partiendo del todo para entender las distintas partes que componen ese todo. El hemisferio holístico es intuitivo en vez de lógico, piensa en imágenes y sentimientos. Este hemisferio emplea un estilo de pensamiento divergente, creando una variedad y cantidad de ideas nuevas, más allá de los patrones convencionales. El currículum escolar toma en cuenta las habilidades de este hemisferio para los cursos de arte, música y educación física.

No siempre el hemisferio lógico se corresponde con el hemisferio izquierdo ni el holístico con el derecho. En principio se pensó que así era, por lo que se determina alumnos HI (o alumnos analíticos) y alumnos HD (o alumnos relajados o globales). Un hemisferio no es más importante que el otro: para poder realizar cualquier tarea se necesita usar los dos, más si es una tarea complicada. Para un aprendizaje eficaz se requieren los dos hemisferios, pero se tiende a usar uno más que el otro, o preferimos pensar de una manera o de otra. Cada manera de pensar está asociada con distintas habilidades.

El aprendizaje de los alumnos en el aula va a variar en función del modo de pensamiento que prefieran. El actual sistema escolar privilegia el hemisferio lógico sobre el hemisferio holístico (los currículum dan mucha importancia a la matemática y la lengua, se privilegia la rapidez para contestar). Además, muchos profesores tuvieron éxito personal con un estilo verbal, secuencial y lógico, y asumen que esto funciona para todos los estudiantes. Interesa organizar el trabajo en el aula, de forma que se potencie la utilización de ambos modos.

\section{IV.EL MÉTODO BOTETANO Y LA TEORÍA DE LOS HEMISFERIOS}

El método que parecía una serie de ejercicios aparentemente inconexos, ahora con la exposición de la teoría de los hemisferios, apuntaba en el mismo sentido: a un aprendizaje de manera holística, apelando a los beneficios que brinda el HD y luego el HI. En una palabra, integrando el uso de los dos hemisferios.

Antes de aplicar el método, se había enseñando apelando fundamentalmente al HI de las habilidades analíticas, verbales y lógicas; no se había explotado todo el enorme potencial del HD, que provee al alumno de muchas experiencias sensoriales y que además posee la ventaja adicional de procesar una mayor cantidad de ítems en forma simultánea, a través de múltiples canales sensoriales. El método encuentra el fundamento de su eficacia en la teoría de la interacción de los dos hemisferios. El método, al enseñar las matemáticas a los niños, logra "encender" el HD, en virtud de estímulos sensoriales, relatos y otros. Se logra despertar el interés, la curiosidad y, por ultimo, "el enganche" del niño con lo que se desea enseñar. Luego, se establece la conexión con el HI. De tal manera que trabajen ambos hemisferios. Esto hace que el método produzca un nivel de aprendizaje verdaderamente extraordinario. 
Con el método, el aprendizaje mental de sumas y restas usando fichas de colores y no dígitos demuestra que es más rápido y mejor que trabajando de manera escrita.

Recordar hasta quince o más cartas y luego sumarlas (nemotécnica. Ejercicio con los niños del Labarthe) creo que se hace con el HD. Cuando en México se les hacía contar a los niños y luego sumar "contextualizando" el ejercicio con una historia y un juego, también se vinculaba claramente con el HD. Los tableros que desarrollé, basados en la yupana inca, también apelan al estímulo visual y táctil, vinculados al HD. Mi técnica para "engancharse" con la lectura también tiene una clara inclinación en el HD. Las vivencias que, por un sentido u otro, llegaron a quedarse grabadas de una manera indeleble en nuestra conciencia no son un claro ejemplo de lo que digo. Permítanme citar, a manera de ejemplo, aquellos geniales párrafos de Proust al respecto: "En el mismo instante en que ese sorbo de té mezclado con sabor a pastel tocó mi paladar... el recuerdo se hizo presente... Era el mismo sabor de aquella magdalena que mi tía me daba los sábados por la mañana. Tan pronto como reconocí los sabores de aquella magdalena... apareció la casa gris y su fachada, y con la casa la ciudad, la plaza a la que se me enviaba antes del medio día, las calles..." Así da inicio a su memorable novela "En busca del tiempo perdido". Un estímulo sensorial (grabado con fuego en su hemisferio derecho) que nunca pudo olvidar. He allí la clave y la respuesta para lograr despertar en los niños un aprendizaje completo y total.

\section{CONCLUSIONES}

1. Un ejemplo me permite culminar este ensayo y llegar a su conclusión: un niño anterior de 8 años, al pedirle que sume mentalmente $9+7$, se sentía abrumado y recurría a los dedos y hacía un conteo lento y engorroso, que muchas veces lo llevaba al error. Entonces decidí mostrarle unas fichas rojas y amarillas. Le dije que las rojas representaban el número 5 y las amarillas el número 1. Luego le pedí que formara primero el número 9 y luego el 7. Se dio cuenta de que ambos números usaban 1 ficha roja y 4 y 2 amarillas, respectivamente. Luego hicimos una sucesión de sumas diferentes $(8+7,6+5,9+5$, etc.). Con un poco de ejercicio se dio cuenta del proceso. Entonces le pedí que ahora no trabaje con fichas, sino que la imagine. Fue increíble cómo en poco tiempo lograba hacer sumas que antes se le hacía muy difícil. Allí me convencí de que el aprendizaje visual, basado en el HD, estaba muy asociado al cálculo mental.

2. Así, se puede concluir que si el aprendizaje se basa casi exclusivamente en el HI, encargada de las habilidades lógicas y verbales y no se trabaja con intensidad con el HD, que opera lo visual, la intuición, la imaginación y la creación, se está dejando de lado una herramienta de aprendizaje muy poderosa. Y por la aplicación del método, que conecta el HD al HI en la enseñanza de las matemáticas, a cuatro mil niños, en el Perú, Bolivia y México, durante una década, se ha podido llegar a resultados extraordinarios. 
LA TEORIÁA DE LOS HEMISFERIOS CEREBRALES Y EL MÉTODO BOTETANO

\section{REFERENCIAS DEL SISTEMA BOTETANO EN LA WEB}

El sistema empezó con el nombre de Multilúdica en Perú y Bolivia. Luego fue registrado en México como Phi Master Kid. Finalmente se ha consolidado como método Botetano.

\section{Como multilúdica (videos de Perú y Bolivia)}

Perú: http://www.youtube.com/watch?v=QOCo1VUgZC8

Bolivia: http://www.youtube.com/watch?v=9hirA9h6iEM.

Artículos publicados en el sitio web llamado artículos $\mathbf{Z}$

http://www.articuloz.com/authors/25352

\section{Video de México}

Aprenden a multiplicar niños de México. http://www.youtube.com/ watch? $=$ Yw2gXH5mdMI

\section{Entrevistas con el Sr. Jaime de Althaus}
A. 12ABR 2013 TV8 CÉSAR BOTETANO, CREADOR DEL SISTEMA
http://www.youtube.com/watch?v=r2XPAi8qi84
B. CÉSAR BOTETANO Y ALUMNOS
http://www.youtube.com/watch?v=baJVTSYHpo8

\section{Telesur de Argentina}

Método Botetano logra un éxito de 95\% en el aprendizaje de matemáticas

http://www.youtube.com/watch?v=_Bk9M28rY0g

\section{Telesur Venezuela}

Revoluciona maestro peruano la forma de aprender a multiplicar

http://www.youtube.com/watch? $\mathrm{v}=\mathrm{v} 46 \mathrm{KmBhRVn} 8$

\section{Derrama Magisterial del Perú}

A. Derrama Noticias

El primer piloto se realizará en la I.E.E. Pedro A. Labarthe de La Victoria http://www.derramanoticias.com/videos.php?cod $=190$

B. Derrama magisterial presenta el Método Botetano en la IEE. Pedro a. Labarthe http://www.youtube.com/watch?v=NMv1YmJVtFo

C. Derrama Magisterial auspicia método Botetano http://www.youtube.com/watch?v=gBD7F6uQYAY 


\section{Entrevista en Radio Exitosa con Claudia Cisneros}

Método para aprendizaje de las matemáticas

http://www.youtube.com/watch?v=ORr_VcJbEas

1. Video del Cusco (implicación respecto alaprendizaje de niños quechua-hablantes)

2. Información de la enseñanza en Huacho

Una referencia de nuestro trabajo en Cusibamba y Ccorca, Cusco 